\title{
Visual Exploration of Time-oriented Patient Data for Chronic Diseases: Design Study and Evaluation
}

\author{
Alexander Rind ${ }^{13}$, Wolfgang Aigner ${ }^{13}$, Silvia Miksch ${ }^{13}$, Sylvia Wiltner ${ }^{2}$, \\ Margit Pohl ${ }^{2}$, Thomas Turic ${ }^{3}$, and Felix Drexler ${ }^{4}$ \\ 1 Institute of Software Technology \& Interactive Systems, \\ Vienna University of Technology, Austria \\ \{rind, aigner, miksch\}@ifs.tuwien.ac.at \\ 2 Research Group Human Computer Interaction, \\ Vienna University of Technology, Austria \\ margit@igw.tuwien.ac.at, s.wiltner@aon.at \\ 3 Department of Information and Knowledge Engineering, \\ Danube University Krems, Austria \\ 4 Landesklinikum Krems, Austria
}

\begin{abstract}
Medical care, particularly for chronic diseases, accumulates a huge amount of patient data over extensive time periods that needs to be accessed and analyzed accordingly. Information Visualization methods hold great promises in turning data deluge into improved quality of medical care. Yet, patient data management systems mostly provide documents, form-based displays, or static visualizations. We present a design study of an interactive visualization system, called VisuExplore, to support long-term care and medical analysis of patients with chronic diseases. VisuExplore offers interaction techniques for effective exploration of time-oriented data and employs simple, but intuitive visualization techniques. It was developed in close cooperation with physicians. We conducted two user studies with nine physicians and 16 students, which indicate that our design is useful and appropriate for particular tasks.
\end{abstract}

Keywords: Information Visualization, time-oriented data, medical information systems, visual exploration, interaction techniques, user study.

\section{Introduction}

Modern medicine puts a huge amount of patient data at the attending physicians' disposal. Particularly in chronic disease care, data accumulates over extensive periods of time and physicians need to access and analyze it accordingly. Ongoing data integration efforts might bridge the technical gap between different data stores but not the gap between data stores and human expertise. In an effort to turn data deluge into improved quality of medical care, we present a design study of an Information Visualization (InfoVis) system to support physicians in exploring time-oriented health care data of patients with chronic diseases. 
Time and time-oriented data play an important role in health care, which is underlined by the definition of the electronic health record (EHR) as "the complete set of information that resides in electronic form and is related to the past, present and future health status or health care provided to a subject of care" [24]. In care for patients with chronic diseases and for analysis of such care, physicians often need to consult these records. While typical queries can be answered quickly, there are often situations that require deeper understanding of the recorded information (e.g., co-development of biometric variables, or how a change of therapy has affected patient status). In addition, physicians need to analyze multiple variables of different data types and irregular sampling. For such exploratory tasks, where they do not have a direct question but learn about the patient in their search, InfoVis holds great promise [10].

Current patient data management systems (PDMS), however, provide only a small part of what InfoVis has to offer for exploring time-oriented data. Often, patient data is available only in electronic documents or PDMS forms that show one examination at a time. This makes it hard to follow patient trends. Visualizations, if provided, are mostly static and do not allow powerful interactions. Furthermore, existing research for patient data visualization has engaged in different design studies that cannot be immediately transferred to our domain problem. These designs either focus on a single type of data (e.g., intervals) or special scenarios, like intensive care, which is mostly concerned with high-frequency, short-term data (cp. Sect. 2).

In close cooperation with physicians at a regional hospital, we have developed task-specific interaction and visualization methods for the diabetes outpatient clinic, called VisuExplore. Through our design, they can explore time-oriented data of a patient with chronic diseases in a coherent user interface. In order to represent variables of different structure, we visualize each variable or each group of homogeneously structured variables in a separate diagram. To provide a frame of reference, these diagrams are aligned to a common time axis. Tackling irregular and independent sampling, we draw items where they fit on the time axis. The use of simple and easy-to-understand visualization methods was requested by users and should make it easy for them to read and interpret the data. This is accompanied by an extensive set of interaction techniques that allows for flexible and effective exploration of the data. Providing a reasonable combination of simplicity and flexibility is a non-trivial achievement.

In general it is advisable to evaluate InfoVis systems with their target users. However, physicians at our partner hospitals have been very busy and we could recruit nine physicians for the usability investigation of VisuExplore. To complement this investigation, we repeated the user study with 16 students. With this combined approach we could back up the result and gain additional insights.

Contribution This paper's primary contribution is a design study of InfoVis methods for medicine. Design studies have been characterized very well by Munzner [20], who also called for more work of this type. Following this, we describe the diabetes outpatient clinic as accompanying domain problem and the user 
requirements guiding our work (Sect. 3). Next, we present the visual encoding and interaction design of our approach (Sect 4$).{ }^{5}$ In a case study we demonstrate the applicability of our approach (Sect. 5) and we report a user study with 16 students on its usability (Sect. 6). For a secondary contribution, we compare the results of this user study with a previous user study conducted with nine physicians [23]. Finally, we draw our conclusions and outline directions for future work (Sect. 7).

\section{Related Work}

There is growing interest in time-oriented data [2] with medical applications being tackled in some prominent design studies.

One group of designs works exclusively on quantitative patient data (e.g., heart rate). Typically, they encode each variable in a scatter plot or a line plot with time on the horizontal axis. The Time Line Browser [8] is a seminal work for the visual exploration of time-oriented patient data in general and this group of designs in special. It defines a solid theoretical foundation and powerful interaction techniques for time-oriented data but its design is generic and not aimed to be easy to use as the paper concentrates on underlying concepts. The Graphical Summary of Patient Status (GSPS) [25] used fine-tuned scatter plots for a high data-ink ratio and arranged them as small multiples. However, this design was intended for printing and is neither interactive nor flexible. The Medical Information Visualization Assistant (MIVA) [9] applies some features of GSPS' scatter plot and integrates them in an interactive design that allows flexible arrangement of medical variables. However, in contrast to our work it is limited to quantitative data and has scatter plots as its only visualization technique.

A second group of designs focuses on nominal patient data (e.g., period of hospitalization) and typically uses a visual representation similar to a Gantt chart, which shows items as bars parallel to a horizontal time axis. For this group, LifeLines [22] is a ground-laying design. Items are grouped to facets, which can be collapsed or expanded. The facets are comparable to our diagrams, but they cannot be rearranged and resized as flexibly. Patient History in Pocket (PHiP) [3] adapts this approach for a mobile device with limited display size and interaction capabilities. Zhang et al. [36] use vertical bars for the 'when' aspect in their design, which they structure based on the 5 -W of journalistic reporting (who, what, where, when, why, and how).

There are other designs for patient data, which do not use a time axis. For example, VIE-VISU [15] provides a metaphor graphic of quantitative patient status over a time interval and repeats this graphic to show changes over time. Sundvall et al. [32] present a design that shows nominal data as placemarks on a zoomable map of the human body. They use animation to show development over time. The 5-W design of Zhang et al. [36] also contains a view of a stylized

\footnotetext{
5 This extends a short paper [28] written at an early stage of the project, which contained the overall concept and requirements analysis but no system design in detail and no user studies.
} 
human body for its 'where' aspect. As a third view 5 -W provides a directed graph of events in the patient record for the 'why' aspect. These two views, however, show only the current patient status, but not development over time.

Comparing patient data to medical guidelines is a related domain problem and has yielded some designs that combine features from the groups of MIVA and LifeLines (e.g., CareCruiser [11], Midgaard [4]). However, these designs cater more to different tasks than open-ended exploration. In the surroundings of this work we also find the problem domains of querying patient databases (e.g., LifeLines2 [34], Similan [35]) and exploring patient cohorts (e.g., CareGiver [5], Dare [7], Gravi++ [14], TimeRider [27], VISITORS [16]).

Overall, VisuExplore stands out due to its flexibility and task-specific interactions for the exploration process. The exploration designs described above only work with either quantitative or nominal data and rely on a single visualization technique that shows development over time. Our design's interactions include most notably the flexible arrangement of variables, smooth zooming and resizing, measuring time spans, and keeping track of time and relevant items.

\section{Domain Problem}

In this section, we lay a foundation for our design study by describing the user requirements and a medical scenario that has accompanied our design process.

\subsection{Methods for User-Centered Design}

This work is embedded into a larger research project studying the application of InfoVis to medical care. We adopted a user-centered design approach [29] and involved potential users from the beginning. We conducted systematic requirements gathering through semi-structured interviews with five physicians at a regional hospital to assess how InfoVis could make their work easier and what kinds of mapping would be appropriate for their data. Furthermore, we chose a concrete medical scenario that would guide the design process and user studies. We used interviews and contextual observation of three patient encounters in the diabetes outpatient clinic to familiarize ourselves with the medical scenario.

We developed the design based on state-of-the-art InfoVis principles. The design underwent an iterative refinement process to gather expert reviews from InfoVis and human-computer interaction specialists in our team. In addition, we used prototypes, first on paper, then implemented in Java using prefuse [12], to test our approach informally with three physicians and a nurse, who are familiar with managing diabetes. These iterations culminated in a case study and a structured evaluation with nine physicians and 16 students, which we present later in this work.

\subsection{Medical Requirements}

Based on the interviews with the physicians we gathered the following user requirements: 
- Simple user interface: The physicians need to gain quick and unambiguous insights. Thus, the interfaces and visualizations should be particularly clear and simple to use.

- Flexible for various medical variables: A single fixed configuration is not sufficient, because different patients or different medical disciplines require the analysis of different sets of variables.

- Time-oriented data: Various measurements of variables over time need to be followed. Development and co-development are of interest.

- Interactivity: A variety of interaction techniques such as (semantic) zooming, resizing of visualizations, grouping, opening medical documents from the visualization, and writing annotations should be included.

- Multiple patients: Some tasks require the comparison and filtering of data of patient cohorts, for example finding out whether some therapies are more effective than others. ${ }^{6}$

\subsection{Medical Scenario}

Diabetes mellitus is a chronic condition in which the human body is no longer capable of managing its glucose (blood sugar). To mitigate this, patients need to change their lifestyle, take oral medication, and/or inject insulin. Otherwise, they are at risk of many complications, e.g., cardiovascular disease, retinal damage, or diabetic coma. The choice of treatment depends on many factors including diabetes type, severity, comorbid diseases, and the patient's experience in keeping their glucose in the optimal range.

In this scenario, we take the point of view of a physician at the diabetes outpatient clinic. Examinations at the clinic are scheduled in intervals between six weeks to three months, depending on the patient's condition. The data set encompasses quantitative data (e.g., fasting blood glucose level, body mass index), nominal point data (e.g., concomitant medication to lower blood pressure), and nominal interval data (e.g., insulin therapy). However, some of the variables are optional and physicians only record them, if they expect relevant changes. In contrast, some other variables such as blood pressure or cholesterol level might also be recorded between two diabetes examinations by another department of the hospital. The data originates from two separate systems: a laboratory information system and a computerized physician order entry system. Together these factors make a good case for how relevant heterogeneous time-oriented data is in practice.

Physicians working for the diabetes outpatient clinic are interested in whether a patient's condition improves or worsens. They want to relate an improvement of some variables to the development of other variables, for example, losing weight and lower glucose. They need to know which types of therapy a patient already had and how these affected medical tests.

\footnotetext{
${ }^{6}$ In the following design process we came to the conclusion that we can support this requirement, analysing the development of multiple patients over time, better through a separate design study, which we present elsewhere [27].
} 


\section{Description of VisuExplore's Design}

Next, we explain how our proposed interaction and visualization techniques (see Fig. 1) meet the domain problem presented above. For this, we set these techniques in context with state-of-the-art InfoVis research and use anonymized medical data as illustrative example.

\subsection{General Layout}

For efficient visualization of time-oriented data, research in InfoVis methods and human perception has yielded a considerable body of guidelines (e.g., [6,33]). However, there is no single visualization technique that can efficiently encode all types of time-oriented data needed for exploration of diabetes data (cp. [2]). Thus, we work with a collection of well-established visualization techniques, each of which can handle one class of time-oriented variables well. We combine diagrams of these techniques in a multiple-view visualization, where all views share the common time axis. Using the perceptually efficient encoding "position on a common scale" for the time aspects of all items of all variables allows the users to keep a common frame of reference. This makes it easy to find out about sequence, co-occurrence, and co-development of multiple, possibly heterogeneous variables. Thus, we accommodate for the important role time plays in fulfilling the tasks of this medical scenario (see Sect. 3.3). For the users, the time axis is visualized using the Gregorian calendar (see Fig. 1.v0). Vertical grid lines that extend from the time axis help users to look up time points of interest.

\subsection{Visualization Techniques}

VisuExplore provides four simple, but intuitive visualization techniques (see Fig. 1): A line plot and a bar chart for quantitative data as well as an event chart and a timeline chart for nominal variables.

Line plots and bar charts are well known and users can interpret them without training. Thus, we meet the design requirements for visualization that are simple to use and to interpret. The bar chart can be used, when individual values are of interest or should be compared. The line plot connects consecutive observations visually and, thus, can be used to spot and compare trends and patterns. The line plot uses a y-axis that scales automatically to a default value range, which is defined in the metadata. Alternatively, users can set it to scale fitting for the highest and lowest values in the patient's data. This allows users to follow changes that are small compared to the value and immediately determine the value range of a variable. However, this setting might lead to wrong insights as small fluctuations of a stable parameter may look like dramatic changes. Further, the y-axis may be set to extend to zero. This is particularly useful when ratios are important (e.g., body weight), but not for interval scaled variables (e.g., temperature). The bar chart uses the same y-axis but it will always extend to zero, because otherwise bar length would not be interpreted correctly. 


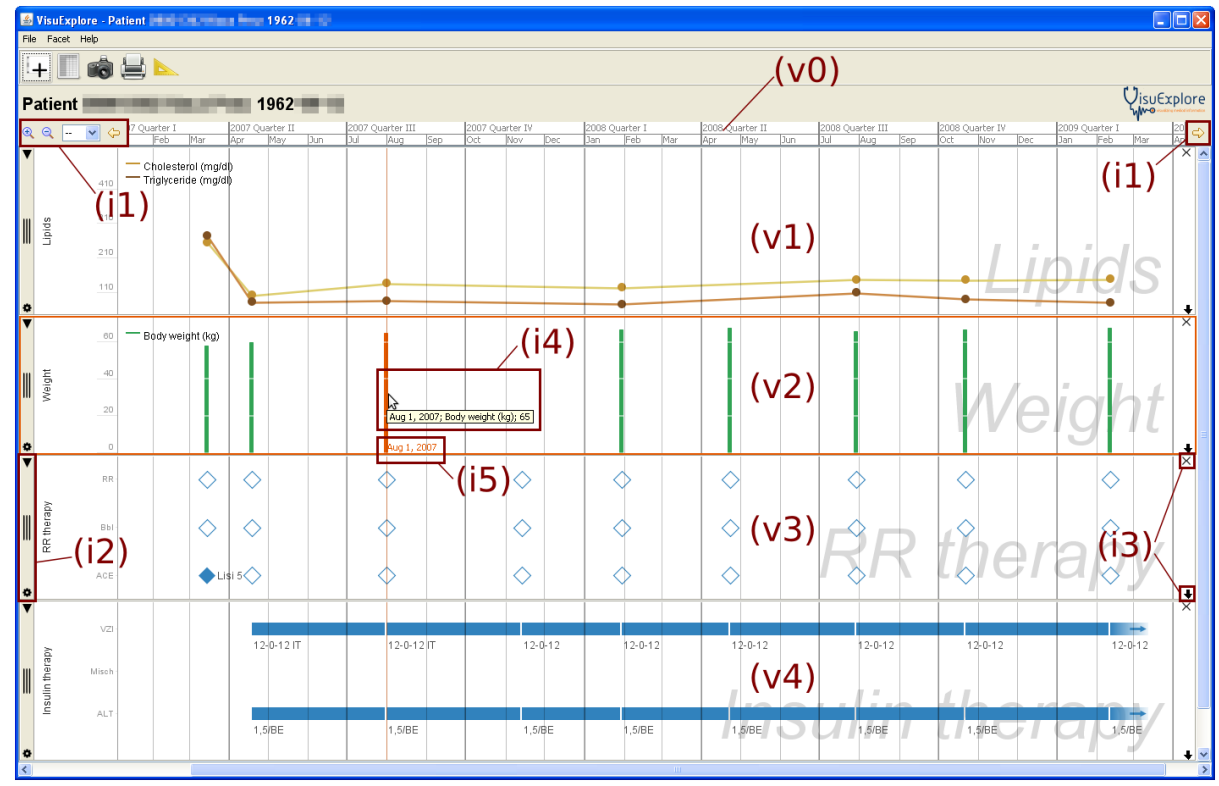

Fig. 1. Exploring medical data of a diabetes patient using the VisuExplore interface: Multiple groups of variables are visualized with different techniques in a multiple views display: Line plot (v1), bar chart (v2), event chart (v3), and timeline chart (v4). All visualizations share a common time axis (v0). Patient master data is printed above the time axis. Users can interact with the visualization through mouse actions inside each view, scrollbars, a toolbar for time (i1), two toolbars for views (i2, i3), and the menu. When hovering over an item, a tooltip (i4) prints the item's exact time and value. A mouse tracker (i5) marks the position of the mouse pointer with an orange line to keep users informed about items occurring together and their occurrence time.

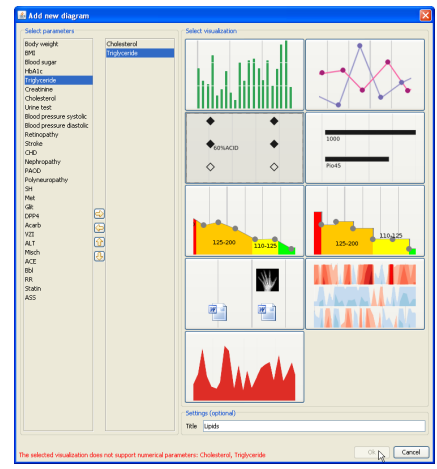

Fig. 2. Dialog to add a new diagram: Users select variables, a technique, and optionally a diagram title. The dialog will warn users, if they choose variables that are not supported by the selected technique. In the user study we used only bar chart, line plot, event chart, and timeline chart (top four options). The advanced visualization techniques were not visible in the user interface. 
For nominal data, we provide the event chart. It can visualize several variables in the same diagram. Each variable is positioned on a separate y-position. Basic shapes represent all variable observations and different nominal values are mapped to the shape or color. For example, in Fig. 1.v3 prescribed drugs are shown as filled diamonds whereas empty diamonds appear for drugs that are not prescribed. A short free text may go along with each of these shapes. In the figure they show an abbreviation of the brand name and the dosage.

So far, we presented visualization techniques for data that is valid for a point in time. When data of a nominal variable is valid for a time interval, we can use the timeline chart (see Fig. 1.v4). This chart type is a well-established technique and can be found in the group of designs around LifeLines [22]. We also devised a representation for intervals of which only the start time is known but not the end time (e.g., currently prescribed insulin therapy). We draw these intervals with the right edge fading out and an arrow should indicate that the timeline might extend longer than it is visible.

The collection of visualization techniques outlined above is not intended to be complete. Instead, we plan VisuExplore as a framework that is open for pluggable components as requested by Aigner et al. [2]. In fact, we have already integrated five advanced visualization techniques to demonstrate the extensibility of VisuExplore (see Fig. 2): a semantic zoom chart (cp. [4]), a step chart, a silhouette graph, a horizon graph (cp. [26]), and a document browser. However, in the usability investigation we limited the prototype to simple techniques, because the introductory requirements analysis called explicitly for simple visualizations. In order not to overwhelm users we plan to evaluate advanced visualization technique one at a time.

\subsection{Interaction Techniques}

Above we discussed visualization techniques but much more is possible through interaction. Interaction is regarded as "at the heart" of InfoVis [31] and this might be even more true for exploration of time-oriented data.

A flexible and adaptable arrangement of variables was one primary requirement for VisuExplore (Sect. 3.2). Our design excels in allowing users interaction with variables. In this respect, it is more powerful than comparable approaches. Users can add new diagrams using a dialog window (see Fig. 2) where they choose variables and a visualization technique.

Each diagram is furnished with two toolbars, one on the left side (see Fig. 1.i2) and one on the right (see Fig. 1.i3), which allow users to adapt the diagram. The $\mathrm{x}$ icon in the top right corner closes the view and removes it from the display and the gearwheel icon in the bottom left corner opens a dialog window that controls settings of the chart (e.g., extending a numerical axis to zero). Furthermore, the toolbars allow users to resize the diagram depending on whether they want an overview by seeing many variables at once or analyze a few in details. They can collapse or expand the diagram to an outline with the triangle icon, which is used in multiple designs (e.g., LifeLines [22]). Alternatively, users can resize the diagram continuously by dragging the arrow icon or the bottom border of 




Fig. 3. Users can move a diagram by clicking and dragging the area indicated through three vertical lines in the left toolbar. While moving, the mouse drags a semi-transparent preview of the diagram and a blue bar marks the current insert location. Here the user moves "HbA1c" (green line plot) up, which will be inserted between "Blood pressure" and "Lipids".

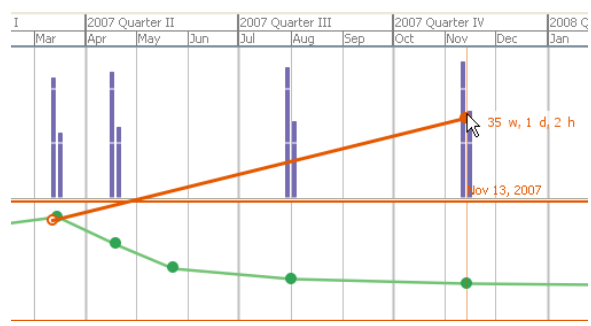

Fig. 4. A measure tool allows users to determine the time interval between any two locations inside the visualization. Here the user finds out that a high blood pressure value comes about 35 weeks after a peak $\mathrm{HbA1c}$ reading.

the diagram. The chart dynamically redraws during interaction and adapts to its height through optimized layout algorithms. By grabbing a diagram in the middle of the left toolbar, users can move diagrams and thus rearrange the configurations (see Fig. 3).

For navigation in time, users can use the pan and zoom buttons in a dedicated toolbar next to the time axis (see Fig. 1.i1), shift the horizontal scrollbar, or drag the mouse in the visualization. There are also some predefined zoom steps, like one week or one year, which users can select in the toolbar. These replicated interactions make navigation usable for both beginners and more experienced users. During zoom, the time axis automatically adapts itself to use time units fitting the current temporal resolution, for example quarter of a year and month (see Fig. 1.v0). With the mouse tracker users can see, which observations occur together and at what time. This is implemented as an orange vertical line that follows the mouse inside the visualization (see Fig. 1.i5). A label accompanies the line and prints the time of its x-coordinate.

An important task when interacting with time-oriented data is to measure the duration of an item or the time difference between two items. Especially in exploring a medical record and in investigating possible cause-effect relationships, time differences between items of different variables need to be studied (e.g., does a change in insulin therapy result in a decrease of HbA1c within a certain amount of time). For this, we created the measure tool (see Fig. 4). Users can determine the time difference from a reference point to the current mouse location through a straight line and a label. The label is updated with every mouse movement and prints the time difference in reasonable time units. This works across multiple diagrams. Similar measure tools are common for spatial data (e.g., Google Maps), but we are not aware about such a functionality in other visualization approaches for time-oriented data. 
Finally, users can interact directly with visual items representing entries in the data set. On mouse-over, an item is highlighted and a tooltip with its exact time and value pops up (see Fig. 1.i4). Users can also mark one or more individual items as selected by clicking on them. Further, users can open an additional window with a table containing the items of one diagram (see Fig. 5). Also in this table, users can select items - individually or block-wise. These items are then marked in the diagram and vice versa, which allows users to interact with one and watch the effects in the other. Users may also sort the items by their value or their occurrence time. Thus, it is possible to highlight, for example, the top five observations of a quantitative variable.

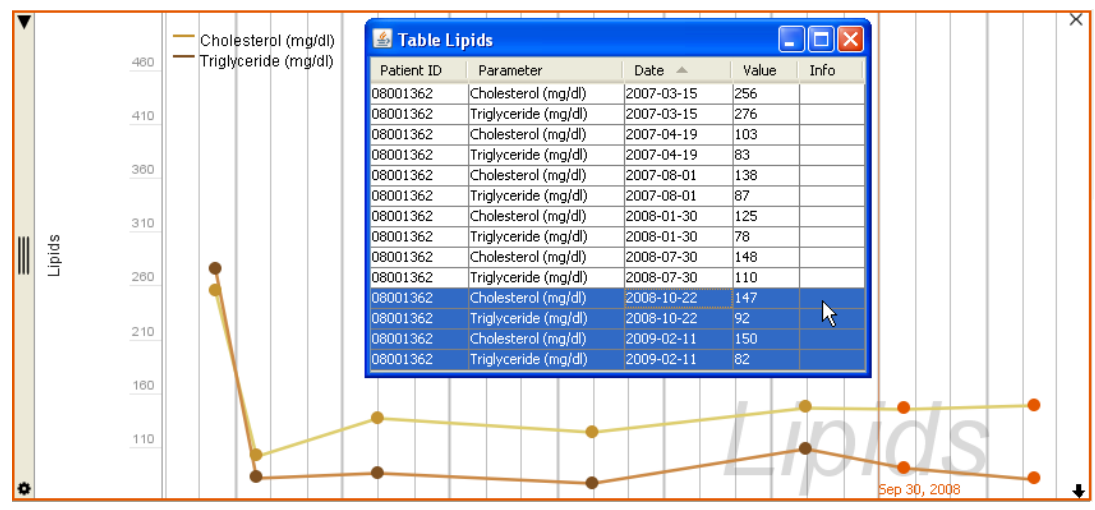

Fig. 5. The table window allows users to inspect all items in detail and sort them by each column. They can select items (color orange) by clicking. Diagram and table are linked, selecting an item in the table will select the corresponding item in the diagram. Here, the user has selected the four latest readings in "Lipids".

\section{Case Study}

In this section, we demonstrate our design in context of a concrete diabetes case that we obtained from our medical project partners. The insights reported here came up, while physicians tested our prototype.

\subsection{Diagnosis of Latent Autoimmune Diabetes of Adults}

In Fig. 6 we show a screenshot of our prototype applied to medical data of a diabetes patient. First, we have created eight diagrams and repeatedly rearranged them so that they provide a good overview of the patient's diabetes history: On top, there is an event chart of end organ damages that can be related to diabetes. Next, we added three line plots with body mass index (BMI), blood glucose, and HbA1c (an indicator for a patient's blood glucose condition over the 


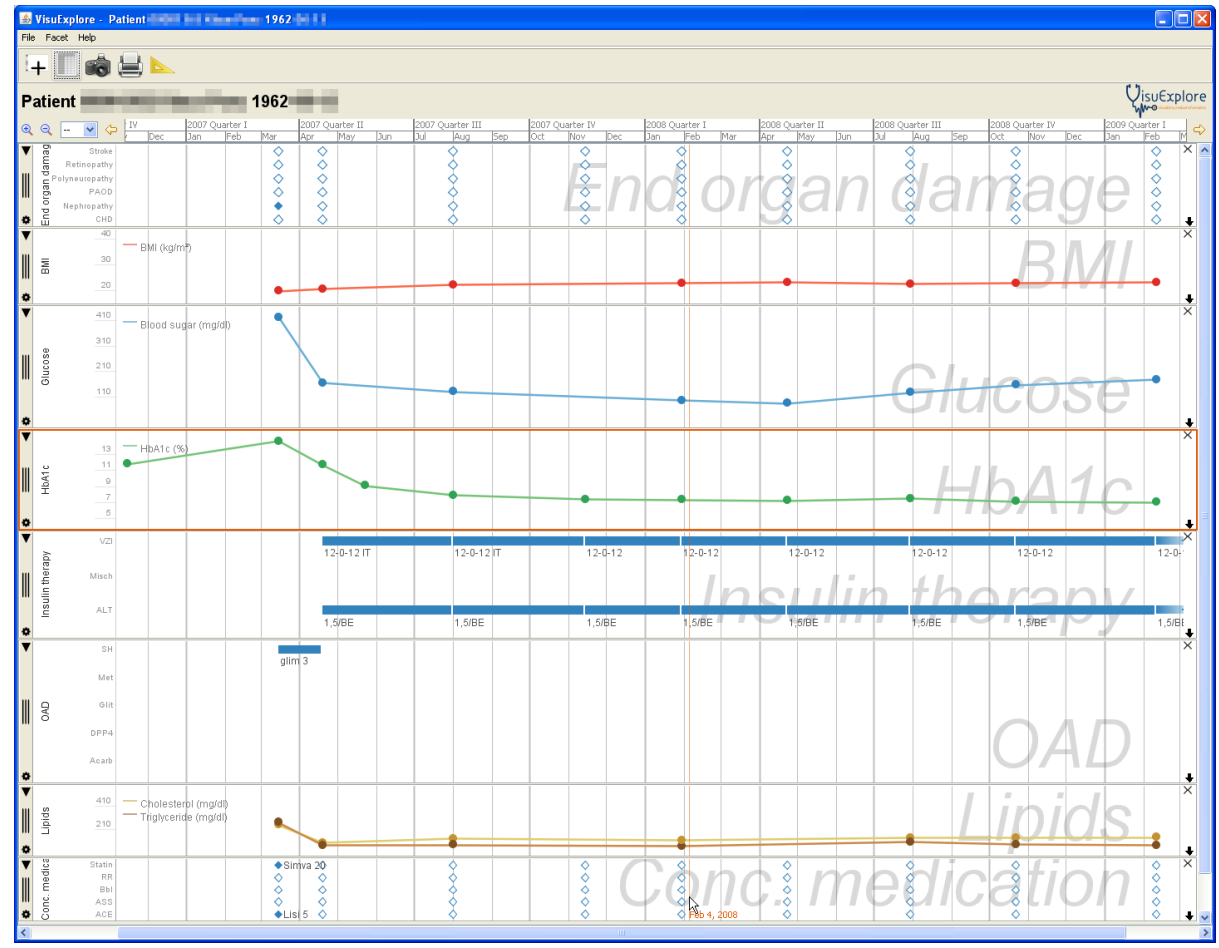

Fig. 6. Patient X (medical data extracted and anonymized in March 2009): Very high blood sugar values and a body mass index of 20.1 at admission are diagnosed as latent autoimmune diabetes of adults. First treatments are "SH glim 3", an oral anti-diabetic drug, with medication for elevated blood pressure and elevated blood lipids. After one month, blood sugar has improved and blood lipids have normalized. Patient switches to insulin therapy and continuously improves.

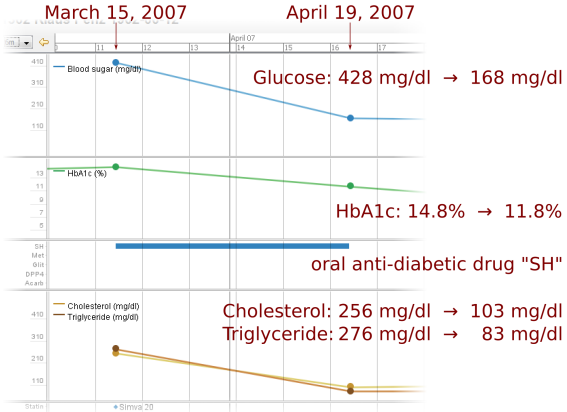

Fig. 7. First and second examination of Patient X show an improvement of blood sugar and blood lipid values



Fig. 8. Detecting local extrema of $\mathrm{HbA1c}$, body weight, creatinine, cholesterol, and triglyceride in the July 2008 examination 
previous several weeks). Below blood sugar values, we placed two timeline charts showing the insulin therapy and oral anti-diabetic drugs. Insulin is categorized into rapid-acting insulin ("ALT"), intermediate-acting insulin ("VZI"), and a mixture of these ("Misch"). Oral anti-diabetic drugs are grouped by five active ingredients/brands. Details about brand name or dosage in free text are shown in labels located under the timeline. The bottom two diagrams are related to blood lipids with a line plot of triglyceride and cholesterol and an event chart of concomitant medication. We chose this arrangement because it places diagrams with medical tests directly above diagrams of the related medical interventions. We reduced the height of some diagrams to fit them on a single screen. Then, we zoomed and panned to a period of two years and three months between November 2006 and February 2009, which shows all examinations.

This diabetes case (see Fig. 6) is a 44-year-old patient with initially very high blood sugar values. We can read the exact values $428 \mathrm{mg} / \mathrm{dl}$ glucose, $14.8 \%$ HbA1c from the tooltips. In addition, blood lipids values are high $(256 \mathrm{mg} / \mathrm{dl}$ cholesterol, $276 \mathrm{mg} / \mathrm{dl}$ triglyceride). At the same time, the body mass index of 20.1 is rather low. Even though there is the suspicion of a nephropathy (damage to or disease of the kidney), these are also signs for latent autoimmune diabetes of adults, a special form of type 1 diabetes.

First treatments are "SH glim 3", an oral anti-diabetic drug, plus medication for elevated blood pressure ("ACE Lisi 5") and elevated blood lipids ("Statin Simiva 20"). After one month, blood sugar has improved (168 mg/dl glucose) and blood lipids have normalized (see Fig. 7). The patient switches to insulin therapy in a combination of rapid-acting insulin ("ALT") and intermediate-acting insulin ("VZI"). We use the measure tool with a reference point in April 2007 and move around to other examinations to get an impression of the time intervals relative to the start of insulin therapy.

Next, we open the tables for HbA1c and BMI because we are interested in exact values although we can see the trend from the visualization as well. We can see that the patient's condition almost continuously improves with a HbA1c value of $7.1 \%$ and a BMI of 23.5 on February 11, 2009. Only on July 30, 2008 , we have a slightly higher HbA1c of $7.6 \%$ and a slightly lower BMI of 22.8 . We want to find out more about July 2008 and add views of other variables: weight, creatinine, blood pressure (see Fig. 8). We notice that on July 30, 2008 also creatinine is higher than usually. A possible explanation for this is that the patient is dehydrated.

The suspicion of nephropathy has been wrong. Since April 2007, the insulin dosage stays stable and concomitant medication is no longer needed. The patient's overall condition has improved through blood sugar management.

In addition to nine examination dates with data on diabetes treatment, our system found two more HbA1c readings in November 2006 and May 2007. A physician involved in the case study wondered about the very high HbA1c value of $11.9 \%$ in November 2006 and why diabetes treatment had only started four months later. Furthermore, the examination in November 2007 lacks data about body mass index, glucose, and lipids. 


\section{Usability Investigation}

\subsection{Description of the Investigation}

The main goal of the investigation was to assess whether VisuExplore was usable and the representations (mapping) of the variables understandable. The subjects were 16 students. The results of this study are compared to another study with the same system and similar methodology (interviews) conducted with nine physicians (see [23]). An additional goal was to assess whether the results from the study with physicians and with students differed considerably. It is often criticized that evaluation studies are conducted with students, but we assume that at least some research questions, especially questions of usability, can also be answered using students as subjects.

The main research questions of the investigation were:

1. Which were the main advantages/disadvantages of the system?

2. Which forms of interaction are most useful?

3 . Which of the proposed visualizations for the variables are appropriate?

4. Are the results of the study with students as subjects similar to the results of the study with physicians (experts)?

The subjects of the study were students. They were tested in two groups. One group (11 students studying computer science) participated in a lecture on Human-Computer Interaction and E-Learning. These students got an introduction of approximately two hours into the application domain and the system. They had to solve three tasks developed by a physician from a hospital participating in the project. They worked on their own with the system and had to fill in a questionnaire with open-ended questions more or less identical to the interview conducted with the physicians [23]. These students had ample time to work with the system - most took about 40 minutes to one hour - and were specifically asked to find usability problems. We decided that 11 students were not enough for the study; therefore we conducted an additional study with five students who studied languages or economics. This had the additional advantage that we did not only test computer science students. The second group received a short introduction into the application domain and system, worked with the system also solving the three tasks - and then had to answer the same questions as the other students in an interview. The questions and tasks for both groups were the same. The only difference was that one group wrote down their answers and the others answered in an interview. The interview enables the researcher to check whether any misunderstandings occurred and to discuss the answers with the subjects. The results from both groups of students did not differ considerably, although the first one produced more usability problems because they worked longer with the system. The longer introduction and the extensive work with the system for the first group probably helped to overcome well-known problems occurring with questionnaires (subjects misunderstanding the questions, lack of contextualization) (see e.g., [1]). 
Both groups got the same three tasks to solve. These tasks were identical to those for the physicians:

Task 1: Please look at the data of three patients (A, B, C), especially at blood sugar, cholesterol and body mass index. Can you see any relationships between the three parameters for these three patients?

Task 2: Look at patient D. Because of an illness, the patient has to take cortisone. What effects does this have on the patient's condition?

Task 3: Take a look at patient E. What can you say about his/her development? (Task 3 is a more general task for exploration).

We used qualitative methods for this investigation because they allow to get detailed information about usability problems in a very efficient manner. In addition, it is possible to analyze unanticipated or controversial usability problems more deeply. Furthermore, recruiting expert users is even harder for a controlled experimental approach. We also used qualitative methods for students because we wanted this study to be as similar to the study with the physicians as possible. In further research, we will also do a quantitative analysis of the log files and the thinking aloud protocols.

The main methods used in this study were a questionnaire with open-ended questions for one group and qualitative interviews for the other group. Questionnaire and interview consisted of the same questions. Sometimes the subjects uttered conflicting statements (e.g., that they appreciated a specific feature of the system, but nevertheless did not use it very often), therefore it is difficult to quantify statements.

\subsection{Results}

In this section, the results of the interviews/questionnaires are described in detail. The emphasis is on comments made by several subjects because we assume that these comments are more relevant for the assessment of the system than a comment made by just one subject. Quotations from the students are added in brackets.

Advantages of the system (research question 1) The answers concerning the main advantages of the system are fairly homogenous. Students noted that the system was easy to learn and intuitive. The interface was easy to handle. There were only a reduced number of features. This made the system easy to understand, but was seen as an advantage and a disadvantage by one subject. The visualization was seen as appropriate. It provides a fast and easy way to get an overview of rather complex data. Different variables can be compared at a glance.

The answers concerning the disadvantages are more heterogeneous, and most of the disadvantages are only noticed by a few subjects. Some of the subjects commented that the design was rather boring ("It looks like Excel."). They also complained that it is not possible to compare the data of two or more patients, which was not in the scope of VisuExplore's design aims. Many of 
them commented that scrolling in some cases was difficult. This was mainly due to the fact that the scrolling function by mouse wheel and automatic scrolling upwards/downwards when dragging a diagram were not implemented in that prototype. Some subjects also noticed that double-click did not work properly. Two of them also complained that there was lack of contrast concerning the colors which made it difficult to distinguish various variables.

In addition, the students found several different smaller usability problems.

Interaction (research question 2) Some of the interaction techniques were appreciated by the subjects while other techniques were criticized:

New Diagram/Delete Diagram: The method to create a new diagram was only appreciated by a few subjects. Several aspects of creating and deleting diagrams were criticized. Some of the subjects had difficulties to create new diagrams for different reasons. Several of the subjects complained that when a visualization technique was not available for a specific variable it was not disabled, but only after selecting the desired technique did an easy to overlook error message appear (cp. Fig. 2). This led to unnecessary user actions. Deleting diagrams also did not seem to be as intuitive as expected. One user, e.g., complained that it was not possible to select more than one diagram to make deletion more efficient.

Resizing Diagrams: Users liked this feature, and there were only a few complaints. It is an important possibility to change between overview and detail-view or to compare a larger number of diagrams.

Moving Diagrams: This feature was explicitly appreciated by eight subjects. They found it useful and well designed. Five subjects pointed out that scrolling and moving diagrams at the same time does not work, which makes this feature inefficient.

Select/Tooltip/Mouse-Over: The attitudes concerning these features were mixed. Six subjects found at least one of these features positive and useful. Four subjects complained that it was not possible to select more than one diagram. The subjects made several different suggestions on how to improve this feature.

Measure Tool: Ten subjects found this feature quite useful because it makes data easier to understand. They made several suggestions how to improve this feature ("rather click at start and end points instead of dragging the line from start to end point").

Pan/Zoom: Only one subject found this feature useful and well implemented. The other subjects criticized it quite heavily. They commented, e.g., that the zoom function does not give a detailed view of the region the subjects were interested in, but some other region. This led to some confusions and disorientation until some (not all) realized that the zoom function zooms into the middle of the time axis and not to the selected data item. Some subjects remarked that the zoom function is not necessary for the data presented in the test.

Visualization (research question 3) The system offered several visualization techniques (line plots, bar charts, etc.). The different possibilities to visualize the 
data were seen as very positive. Most of the visualization techniques are easy to understand. It is simple and intuitive to compare different variables over time. The subjects especially liked line plots and bar charts. The diamond shaped event charts were not understood intuitively and criticized by five subjects.

Most of the students appreciated the possibility to show several variables in one diagram because it makes the comparison of the variables easier ("ideal for up to three variables, more variables would be confusing"; "several lines in one diagram are easier to compare, they do not take up so much space"). No student explicitly criticized this feature.

Comparison to the study with physicians (research question 4) Basically, the results of the study with physicians and the study with students are fairly similar. It should be pointed out, however, that there are a few differences. The results for the question about the main advantages of the systems are more or less the same. Physicians as well as students found that the main advantages of the system are that it is easy to understand and to learn and that the visualization allows the interpretation of the data at a glance. The students found more usability problems, which is not surprising because many of them worked with the system for a longer period of time than the physicians and were specifically asked to identify such problems.

The attitude concerning the creation and deletion of diagrams was quite similar for physicians and students. For both groups we could identify mixed attitudes, some liked this features and some criticized it. Students as well as physicians liked the possibility to resize diagrams, although a few physicians argued that this features is not necessary or even misleading. Moving diagrams was also appreciated by physicians as well as students, although the students more often pointed out that there were technical problems concerning the implementation. Both physicians and students liked the select/tooltip/mouse-over functionality. Again, there are a few usability problems to solve which were noticed by both students and physicians. There are different attitudes concerning the measure tool for physicians and students. The students appreciated this tool much more than the physicians. Both physicians and students were critical concerning the zoom/pan functionality. This is probably due to some implementation problems and also to the fact that especially the zoom functionality is only necessary for large amounts of heterogeneous data. Several subjects (among physicians as well as students) pointed out that they did not really need to zoom to interpret the data provided during the test.

Concerning visualization techniques, physicians had a distinct appreciation for line plots. The students also preferred the line plots, but they also liked the bar charts. Both groups were skeptical about the diamond shaped event charts which they did not find intuitive and understandable. Physicians were more critical about combining several variables in one diagram because the scales of the variables are too different to represent them in one diagram.

In general, the results of the studies for physicians and students are quite similar. There are differences concerning the number of usability problems found 
because many of the students worked longer with the system than the physicians. Students also have a tendency to prefer more novel features (e.g., the measure tools), but it should be pointed out that they also found simple and well-known visualization forms much more useful than complex and new ones.

\section{Conclusions and Future Work}

Interactive InfoVis has the potential to help physicians exploring time-oriented patient data, which is of special relevance in patient-centric care for chronic diseases. We presented VisuExplore, a design study and a prototypical implementation of interactive InfoVis methods to support long-term care and medical analysis of patients with chronic diseases. Designing for such an exploration process, it is essential to consider the medical scenario and user requirements. During our iterative design and prototypical implementation phase, it became clear that our design needs to be flexible and adaptable as well as simple to use and to interpret in order to support the users in their exploration processes. Through combination of easy to understand and mostly well-established visualization techniques (e.g., line plot), powerful interaction techniques (e.g., rearrange diagrams, measure tool), and use of variable metadata we provide a design that meets these requirements.

We demonstrate VisuExplore's applicability using a case study to explore and analyze a patient with a non-typical type of diabetes. Furthermore, we conducted two usability studies, one with nine physicians (cp. [23]) and the other with 16 students, which we reported in this paper. These user studies indicate that our design is useful and appropriate for particular tasks. We have specifically analyzed usability problems connected to interaction techniques. As Lam [17] points out, less than a third of a large number of InfoVis papers she surveyed mentioned interaction explicitly. In doing so, we hope to contribute further to the development of a science of interaction [21]. Comparing the studies of physicians and students we found that the results were fairly similar. Therefore, we conclude that studies with students can be an adequate instrument for usability investigation. Though, for some questions, e.g., on the applicability of a feature, studies with domain experts are needed.

Starting from the work presented here, we see several directions for future research: First, results of the usability investigation point us at possibilities for improvement of VisuExplore. Specifically, we plan to improve the event chart visualization and interactions for panning, zooming, and scrolling. Second, we will do a quantitative analysis of the log files and the thinking aloud protocols. In future user studies, we will also investigate advanced visualization techniques in context of patients with chronic diseases. After adapting them for this concrete domain problem, we want to evaluate if they are more suitable than the simple visualization techniques described above. Third, to explore larger data sets quantitatively and qualitatively, we can apply temporal data abstractions. These visualizations of abstracted data (cp. $[16,19])$ can be integrated directly in our design and allow continuous exploration from overview to detail as we 
demonstrate with the semantic zoom chart. Furthermore, this should allow users to jointly explore variables of significantly different sampling frequency. Finally, our design can provide features to better support humans in an undirected exploration process. For example, users may wish to undo an interaction (e.g., closing a diagram), return to a previous state in their exploration history, or open a predefined set of diagrams (cp. [30]). Other features may afford to externalize hypotheses, which physicians have found during visual exploration, so they can discuss them with colleagues or analyze them statistically (cp. $[13,18])$.

Acknowledgments. This work was supported by the Bridge program of the Austrian Research Promotion Agency (project no. 814316) and the Centre for Visual Analytics Science and Technology CVAST (funded by the Austrian Federal Ministry of Economy, Family and Youth in the exceptional Laura Bassi Centres of Excellence initiative). We wish to thank our partners: NÖ Gesundheitsund Sozialfonds, NÖ Landeskliniken-Holding, Landesklinikum Krems, systema Human Information Systems.

\section{References}

1. Adams, A., Cox, A.L.: Questionnaires, in-depth interviews and focus groups. In: Cairns, P., Cox, A.L. (eds.) Research Methods for Human-Computer Interaction, pp. 17-34. Cambridge University Press, Cambridge, NY (2008)

2. Aigner, W., Miksch, S., Müller, W., Schumann, H., Tominski, C.: Visualizing timeoriented data - a systematic view. Computers \& Graphics 31(3), 401-409 (2007)

3. Ardito, C., Buono, P., Costabile, M.F., Lanzilotti, R.: Two different interfaces to visualize patient histories on a PDA. In: Proc. 8th Conf. Human-Computer Interaction with Mobile Devices and Services. pp. 37-40. ACM (2006)

4. Bade, R., Schlechtweg, S., Miksch, S.: Connecting time-oriented data and information to a coherent interactive visualization. In: Proc. ACM SIGCHI Conf. Human Factors in Computing Systems (CHI). pp. 105-112 (2004)

5. Brodbeck, D., Gasser, R., Degen, M., Reichlin, S., Luthiger, J.: Enabling large-scale telemedical disease management through interactive visualization. In: Connecting Medical Informatics and Bio-Informatics, Proc. Int. Congress Eur. Fed. Med. Inform. (MIE). pp. 1172-1177 (2005)

6. Card, S.K., Mackinlay, J.D., Shneiderman, B. (eds.): Readings in Information Visualization: Using Vision to Think. Morgan Kaufmann, San Francisco (1999)

7. Catarci, T., Santucci, G., Silva, S.F.: An interactive visual exploration of medical data for evaluating health centres. Journal of Research and Practice in Information Technology 35(2), 99-119 (2003)

8. Cousins, S.B., Kahn, M.G.: The visual display of temporal information. Artificial Intelligence in Medicine 3(6), 341-357 (1991)

9. Faiola, A., Newlon, C.: Advancing critical care in the ICU: a Human-Centered biomedical data visualization systems. In: Robertson, M.M. (ed.) EHAWC 2011 and HCII 2011. LNCS, vol. 6779, pp. 119-128. Springer, Heidelberg (2011)

10. Fekete, J.D., van Wijk, J., Stasko, J.T., North, C.: The value of information visualization. In: Kerren, A., Stasko, J.T., Fekete, J.D., North, C. (eds.) Information Visualization, LNCS, vol. 4950, pp. 1-18. Springer, Heidelberg (2008) 
11. Gschwandtner, T., Aigner, W., Kaiser, K., Miksch, S., Seyfang, A.: CareCruiser: exploring and visualizing plans, events, and effects interactively. In: Proc. IEEE Pacific Visualization Symp. (PacificVis). pp. 43-50 (2011)

12. Heer, J., Card, S.K., Landay, J.A.: prefuse: A toolkit for interactive information visualization. In: Proc. ACM SIGCHI Conf. Human Factors in Computing Systems (CHI). pp. 421-430 (2005)

13. Heer, J., Vigas, F.B., Wattenberg, M.: Voyagers and voyeurs: Supporting asynchronous collaborative visualization. Communications of the ACM 52(1), 87-97 (2009)

14. Hinum, K., Miksch, S., Aigner, W., Ohmann, S., Popow, C., Pohl, M., Rester, M.: Gravi++: Interactive information visualization to explore highly structured temporal data. Journal of Universal Computer Science 11(11), 1792-1805 (2005)

15. Horn, W., Popow, C., Unterasinger, L.: Support for fast comprehension of ICU data: Visualization using metaphor graphics. Methods of Information in Medicine 40(5), 421-424 (2001)

16. Klimov, D., Shahar, Y., Taieb-Maimon, M.: Intelligent visualization and exploration of time-oriented data of multiple patients. Artificial Intelligence in Medicine 49(1), 11-31 (2010)

17. Lam, H.: A framework of interaction costs in information visualization. IEEE Trans. Visualization and Computer Graphics 14(6), 1149-1156 (2008)

18. Lammarsch, T., Aigner, W., Bertone, A., Miksch, S., Rind, A.: Towards a concept how the structure of time can support the visual analytics process. In: Miksch, S., Santucci, G. (eds.) Proc. Int. Workshop Visual Analytics (EuroVA 2011) in conjunction with EuroVis 2011. pp. 9-12. Eurographics, Goslar, Germany (2011)

19. Martins, S.B., Shahar, Y., Goren-Bar, D., Galperin, M., Kaizer, H., Basso, L.V., McNaughton, D., Goldstein, M.K.: Evaluation of an architecture for intelligent query and exploration of time-oriented clinical data. Artificial Intelligence in Medicine 43(1), 17-34 (2008)

20. Munzner, T.: Process and pitfalls in writing information visualization research papers. In: Kerren, A., Stasko, J.T., Fekete, J.D., North, C. (eds.) Information Visualization, LNCS, vol. 4950, pp. 134-153. Springer, Berlin (2008)

21. Pike, W.A., Stasko, J., Chang, R., O'Connell, T.A.: The science of interaction. Information Visualization 8(4), 263-274 (2009)

22. Plaisant, C., Mushlin, R., Snyder, A., Li, J., Heller, D., Shneiderman, B.: LifeLines: using visualization to enhance navigation and analysis of patient records. In: Proc. AMIA Symp. pp. 76-80 (1998)

23. Pohl, M., Wiltner, S., Rind, A., Aigner, W., Miksch, S., Turic, T., Drexler, F.: Patient development at a glance: An evaluation of a medical data visualization. In: Campos, P., Graham, N., Jorge, J., Nunes, N., Palanque, P., Winckler, M. (eds.) INTERACT 2011, Part IV. LNCS, vol. 6949, pp. 292-299. Springer, Heidelberg (2011)

24. Potamias, G.: State of the art on systems for data analysis, information retrieval and decision support. Deliverable D13, INFOBIOMED project (2006), http: //www.infobiomed.org/paginas_en/INFOBIOMED_D13_final.pdf, access July 14, 2010

25. Powsner, S.M., Tufte, E.R.: Graphical summary of patient status. Lancet 344(8919), 386-389 (1994)

26. Reijner, H.: The development of the horizon graph. In: Bartram, L., Stone, M., Gromala, D. (eds.) Proc. Vis08 Workshop From Theory to Practice: Design, Vision and Visualization (2008) 
27. Rind, A., Aigner, W., Miksch, S., Wiltner, S., Pohl, M., Drexler, F., Neubauer, B., Suchy, N.: Visually exploring multivariate trends in patient cohorts using animated scatter plots. In: Robertson, M.M. (ed.) EHAWC 2011 and HCII 2011. LNCS, vol. 6779, pp. 139-148. Springer, Heidelberg (2011)

28. Rind, A., Miksch, S., Aigner, W., Turic, T., Pohl, M.: VisuExplore: gaining new medical insights from visual exploration. In: Hayes, G.R., Tan, D.S. (eds.) Proc. Int. Workshop on Interactive Systems in Healthcare (WISH@CHI2010). pp. 149152. SIGCHI (2010)

29. Sharp, H., Rogers, Y., Preece, J.: Interaction Design: Beyond Human-Computer Interaction. Wiley \& Sons, Chichester, second edn. (2007)

30. Shrinivasan, Y.B., van Wijk, J.J.: Supporting the analytical reasoning process in information visualization. In: Proc. ACM SIGCHI Conf. Human Factors in Computing Systems (CHI). pp. 1237-1246 (2008)

31. Spence, R.: Information Visualization: Design for Interaction. Prentice Hall, Upper Saddle River, NJ, second edn. (2007)

32. Sundvall, E., Nystrom, M., Forss, M., Chen, R., Petersson, H., Ahlfeldt, H.: Graphical overview and navigation of electronic health records in a prototyping environment using google earth and openEHR archetypes. In: Kuhn, K.A., Warren, J.R., Leong, T. (eds.) Proc. 12th World Congr. Health (Medical) Informatics (Medinfo 2007). pp. 1043-1047. IOS, Amsterdam (2007)

33. Tufte, E.R.: The Visual Display of Quantitative Information. Graphics Press, Cheshire, CT (1983)

34. Wang, T.D., Plaisant, C., Shneiderman, B., Spring, N., Roseman, D., Marchand, G., Mukherjee, V., Smith, M.: Temporal summaries: Supporting temporal categorical searching, aggregation and comparison. IEEE Trans. Visualization and Computer Graphics 15(6), 1049-1056 (2009)

35. Wongsuphasawat, K., Shneiderman, B.: Finding comparable temporal categorical records: A similarity measure with an interactive visualization. In: Proc. IEEE Symp. Visual Analytics Science and Technology (VAST). pp. 27-34 (2009)

36. Zhang, Z., Mittal, A., Garg, S., Dimitriyadi, A.E., Ramakrishnan, I.V., Zhao, R., Viccellio, A., Mueller, K.: A visual analytics framework for emergency room clinical encounters. In: IEEE Workshop on Visual Analytics in Health Care (2010), http: //research.ihost.com/vahc2010/, access May 5, 2011 